\title{
Impaired function of the intestinal barrier in a novel sub-health rat model
}

\author{
SISI FENG ${ }^{1}$, WEIDONG LIU ${ }^{2}$, SHENGNAN ZUO $^{3}$, TINGYAN XIE ${ }^{1}$, \\ HUI DENG ${ }^{1}$, QIUHUAN ZHANG ${ }^{1}$ and BAIYUN ZHONG ${ }^{1}$ \\ ${ }^{1}$ Department of Clinical Laboratory, Xiangya Hospital of Central South University, Changsha, Hunan 410008; \\ ${ }^{2}$ Department of Clinical Laboratory, Hunan Guangxiu Hospital, Changsha, Hunan 410000; \\ ${ }^{3}$ Hepatobiliary and Enteric Surgery Research Center, Xiangya Hospital of Central South University, \\ Changsha, Hunan 410008, P.R. China
}

Received December 7, 2014; Accepted August 27, 2015

DOI: $10.3892 / \mathrm{mmr} .2016 .4978$

\begin{abstract}
Sub-health is a state featuring a deterioration in physiological function between health and illness, and the sub-health condition has surfaced as life-threatening in humans. The aim of the present study was to establish a sub-health model in rats, and investigate the function of the intestinal barrier in the sub-health rats and rats following intervention. To establish a sub-health model, the rats were subjected to a high-fat and sugar diet, motion restriction and chronic stress. Their serum glucose and triglyceride levels, immune function and adaptability were then measured. The levels of diamine oxidase and D-lactic acid in the plasma were analyzed as markers of the intestinal permeability. The protein and mRNA expression levels of anti-apoptotic YWHAZ in the colonic tissue was detected using immunohistochemical and reverse transcription-quantitative polymerase chain reaction analyses In the present study, the sub-health rat model was successfully established, and sub-health factors increased the intestinal permeability and reduced the expression of YWHAZ. Providing sub-health rats with normal living conditions did not improve the function of the intestinal barrier. In conclusion, the results of the present study demonstrated that intestinal disorders in the sub-health rat model may result from the damage caused by reduce intestinal barrier function as well as the decreased expression levels of YWHAZ. Additionally, rats in the sub-health condition did not recover following subsequent exposure to normal living conditions, suggesting that certain exercises or medical intervention may be necessary to improve sub-health symptoms.
\end{abstract}

Correspondence to: Dr Baiyun Zhong, Department of Clinical Laboratory, Xiangya Hospital of Central South University, 87 Xiangya Road, Changsha, Hunan 410008, P.R. China E-mail: csuzhongbaiyun@163.com

Key words: sub-health, intestinal barrier, rat, apoptosis

\section{Introduction}

Health is a state of complete physical, mental and social well-being, and not merely the absence of disease or infirmity. The sub-health condition refers to a state in which the patient experiences a reduction in his or her vitality and adaptability, although no defined disease is diagnosed (1). It is also a state featuring a deterioration in physiological function between health and illness $(2,3)$. The sub-health condition has emerged as a life-threatening condition in China, which is prevalent among senior and middle-level managers, clerks and other workers of high educational attainment (4). The causes are varied, including poor diet, lack of exercise and chronic stress, which are all important in the development of the sub-health condition. The condition can last for several months or even years, and patients suffering from this condition often cannot work efficiently, or cannot continue their work or studies, thereby affecting their relationship with family members and society (5). Certain experts have summarized the characteristics of the condition as a deficit of activity, reaction and adaptability. In addition, the sub-health condition is characterized by an increase in the levels of blood glucose, lipid and viscosity, and a decrease in immune function (5). Few animal models have been established to investigate the development of the sub-health condition. The aim of the present study was to establish a novel rat sub-health model, according to its causes, and to evaluate the success of the model against the characteristics of the sub-health condition.

Intestinal disorders, including constipation and diarrhea, are the most common symptoms seen in individuals in sub-health. Previous reports have demonstrated that abnormal intestinal barrier function occurs in irritable bowel syndrome, prolonged and high-intensity exercise, and with high levels of dietary fat $(6,7)$, and the mechanism contributes to their intestinal symptoms of diarrhea and constipation. In addition, individuals in sub-health exhibit a notable downregulation in the expression of YWHAZ, which has protective effects on intestinal barrier function (8). Therefore, the present study performed experiments to characterize changes in intestinal function in the novel sub-health rat model. 
There remains controversy as to whether the sub-health condition requires medical intervention, and it has been suggested that removal of the causes, including the intake of high-fat foods and physical stress, can cause sub-health symptoms to disappear. In the present study, the sub-health model rats we re-introduced their normal environment following establishment of the model, following which the physiological index and expression levels of YWHAZ were analyzed. The present study aimed to elucidate possible underlying mechanisms of intestinal disorders.

\section{Materials and methods}

Sub-health rat model. The present study was approved by the Ethics Committee of Xiangya Hospital (Changsha, China). A total of 18 healthy adult male Wistar rats (weighing 180-200 g) were purchased from the animal facility of Central South University. The rats were housed individually, provided with access to food and water ad libitum and maintained under a $12 \mathrm{~h} \mathrm{light} / \mathrm{dark}$ cycle at a temperature of $25 \pm 2^{\circ} \mathrm{C}$. The rats were randomly divided into a sub-health group $(n=12)$ and a control group $(n=6)$.

In the sub-health group, the rats were subjected to high-fat and sugar diet (comprising 15\% lard and 10\% sucrose) in their feed every day. At the same time, the rats were placed in a restrictive space $24 \mathrm{~h}$ a day, which limited motion permitting the rat to turn around. In addition, the rats were subjected to water avoidance stress (WAS) every day. The procedure of WAS involved placing the rat on a platform $(8 \times 6 \mathrm{~cm})$ in the middle of a plastic container $(56 \times 50 \mathrm{~cm})$ filled with warm water $\left(25^{\circ} \mathrm{C}\right)$ to $1 \mathrm{~cm}$ below the height of the platform. The rats avoided the aversive stimulus (water) by remaining on the platform for $1 \mathrm{~h}$. The experimental procedures were performed between 8:00 and 10:00 a.m. to minimize the effect of their circadian rhythm (9). This series of experiments was performed for 5 weeks in total. At the same time, the control group of rats was fed a normal diet. After 5 weeks, six of the rats in the sub-health group and the six control group rats were randomly selected and sacrificed by decapitation, following which trunk blood and $10 \mathrm{~g}$ feces samples, and organs were collected. The colon was dissected and maintained on ice prior to weighing within 2-3 h of collection. Whole blood was allowed to clot and centrifuged at $3,220 \mathrm{x} \mathrm{g}$ for $20 \mathrm{~min}$ at $4^{\circ} \mathrm{C}$ to collect the serum, which was stored at $-70^{\circ} \mathrm{C}$. The remaining six sub-health group rats, were removed from the sub-health condition and returned to their normal living patterns, including a normal diet, sufficiently large cage and lowered levels of stress. After 5 weeks, these rats were also sacrificed and blood and feces samples and organs were collected.

Glucose and lipid profile analysis. The serum was obtained and stored in $0.5 \mathrm{ml}$ aliquots at $-70^{\circ} \mathrm{C}$ until use. The levels of glucose (GLU; cat. no. YZB/GER 3263-2014), triglycerides (TGs; cat. no. YZB/GER 3373-2014), total cholesterol (T-Ch; cat. no. YZB/GER 3316-2014), high density lipoprotein cholesterol (HDL-c; cat. no. YZB/GER 3394-2014) and low density lipoprotein cholesterol (LDL-c; cat. no. YZB/GER 3364-2014) were measured using the corresponding commercial kits (Autec Diagnostica Co, Botzing, Germany) on an automatic biochemistry analyzer (HITACHI 7170, Hitachi, Ltd., Tokyo, Japan).

Serum concentration analysis of interferon (IFN)- $\gamma$ and interleukin (IL)-4. The serum concentrations of INF- $\gamma$ and IL-4 were measured using a two-step sandwich enzyme-linked immunosorbent assay (ELISA) method, with INF- $\gamma$ and IL-4 assay kits, which were purchased from Cusabio (Wuhan, China), in accordance with the manufacturer's instructions.

Open field assessment. The rats were removed from their housing cages and placed at the center of a wooden box measuring 100x100x40 cm, the floor of which was divided by black lines into 25 equal squares. The horizontal locomotion, vertical locomotion, time spent in the central area of the apparatus and the number of defecations were recorded for $5 \mathrm{~min}$, to evaluate habituation, emotionality and motor activity. The horizontal locomotor activity was characterized by the total number of squares crossed during a 5 min assessment session (square crossing), and the vertical locomotion was determined by the number of rearings (standing on hind legs) (10).

Determination of concentration of serum D-lactic acid and activity of diamine oxidase (DAO). The concentrations of serum D-lactic acid concentration was measured using a two-step sandwich ELISA method, with a D-lactic acid kit (Cusabio), according to the manufacturer's instructions. Serum DAO activity was measured using a spectrophotometric method, using D-lactic acid and DAO activity assay kits (Jiancheng Bioengineering Institute, Nanjing, China) in the HITACHI 7170 system.

Histological examination. Samples of the colon were fixed in $10 \%$ buffered paraformaldehyde (Shanghai Qiangshun Chemical Reagents Co., Ltd., Shanghai, China), embedded in paraffin (Shanghai Qiangshun Chemical Reagents Co., Ltd.), sectioned at $4 \mu \mathrm{m}$ thickness, and stained with hematoxylin and eosin (Shanghai Qiangshun Chemical Reagents Co., Ltd.) A Bioquant image analysis system (Bioquant Image Analysis Corporation, Nashville, TN, USA) attached to a Nikon E800 microscope (Nikon Corporation, Tokyo, Japan) was used to visualize the stained sections at three fields per section.

$R N A$ extraction and reverse transcription-quantitative polymerase chain reaction ( $R T-q P C R)$ analysis. Total RNA was isolated from $100 \mathrm{mg}$ samples of colon tissue using TRIzol reagent (Invitrogen Life Technologies, Carlsbad, CA, USA) and reverse-transcribed using a RevertAid First Strand cDNA Synthesis kit (Thermo Fisher Scientific, Pittsburg, PA, USA), according to the manufacturer's instructions. The qPCR reaction was performed in a $20 \mu \mathrm{l}$ reaction volume, containing $1.6 \mu \mathrm{l}$ of primer, using an Applied Biosystems Max3000 sequence detection system and SYBR Green reagents (Takara Bio Inc., Otsu, Japan). The thermocycling conditions were $95^{\circ} \mathrm{C}$ for $10 \mathrm{~min}$, followed by 40 cycles of denaturation at $95^{\circ} \mathrm{C}$ for $35 \mathrm{sec}$ and annealing/elongation at $60^{\circ} \mathrm{C}$ for $20 \mathrm{sec}$. The oligonucleotide primers (obtained from Shanghai Shenggong Biology Engineering Technology Service, Ltd., Shanghai, China) used were as follows: Rat YWHAZ, forward 5'-GCCTGCTCTCTTGCAAAAAC-3' and reverse 
Table I. Effects on the duration spent in the central area, number of rearings, crossings and fecal boli in the open field assessment in rats exposed to sub-health conditions for 5 weeks and subsequent recovery.

\begin{tabular}{lccc}
\hline Variable & Control & Sub-health & Sub-health recovery \\
\hline Duration in central area (sec) & $4.33 \pm 2.50$ & $14.83 \pm 9.45^{\mathrm{a}}$ & $9.17 \pm 8.33$ \\
Number of rearings & $20.83 \pm 10.09$ & $4.33 \pm 3.72^{\mathrm{a}}$ & $12.00 \pm 7.07$ \\
Number of crossing & $66.50 \pm 39.79$ & $18.67 \pm 17.39^{\mathrm{a}}$ & $39.83 \pm 28.48$ \\
Number of fecal boli & $1.33 \pm 1.75$ & $4.00 \pm 2.19^{\mathrm{a}}$ & $2.17 \pm 0.40$ \\
\hline
\end{tabular}

Data are expressed as the mean \pm standard deviation. ${ }^{\mathrm{a}} \mathrm{P}<0.05$, vs. control group (Student $\mathrm{t}$-test).

5'-GGTATCCGATGTCCACAATG-3'; and Rat GAPDH, forward 5'-ATCACCATCTTCCAGGAGCG-3' and reverse 5'-TTCTGAGTGGCAGTGAGGGC-3'. Detection of the qPCR products was monitored by the increase in fluorescence caused by the binding of SYBR Green to the double-stranded DNA. To verify the homogenous nature of the qPCR product, melting-point determinations were evaluated at the end of each reaction. The change in cycle threshold was calculated to compare the mRNA expression levels of each target gene, relative to expression of the reference 'housekeeping' gene, GAPDH. $\triangle \mathrm{Ct}$ indicates the difference between the number of cycles required to detect linear amplification of the PCR products for each protein and that of GAPDH. The relative expression levels were analyzed using the $2^{-\Delta \mathrm{Ct}}$ method.

Immunohistochemistry. The protein expression levels were evaluated using immunohistochemistry. Sections were cut from the paraffin blocks $(4 \mu \mathrm{m})$ and mounted onto silane-coated glass slides (Beijing Zhongshan Jinqiao Biotechnology Co., Ltd., Beijing, China). The sections were dewaxed in xylene (Beijing Zhongshan Jinqiao Biotechnology Co., Ltd.) and rehydrated via a graded series of alcohols. Antigen retrieval was performed using $10 \mathrm{mmol} / 1$ citrate buffer ( $\mathrm{pH}$ 6.0; Beijing Zhongshan Jinqiao Biotechnology Co., Ltd.) and heat, with sections subjected to microwave irradiation at $700 \mathrm{~W}$ and $45^{\circ} \mathrm{C}$ to produce staining. Endogenous peroxidases were inhibited through incubation for $30 \mathrm{~min}$ at $25^{\circ} \mathrm{C}$ with $3 \% \mathrm{H}_{2} \mathrm{O}_{2}$ in methanol. The tissue sections were incubated for $2 \mathrm{~h}$ at room temperature with $3 \%$ normal goat serum prior to incubation with the primary rabbit monoclonal anti-rat YWHAZ antibody (Santa Cruz Biotechnology, Inc., Santa Cruz, CA, USA; cat. no. sc-1019) diluted 1:100 in PBS for $16-24 \mathrm{~h}$ at $4^{\circ} \mathrm{C}$. The bound antibodies were detected using biotin-substance P-conjugated goat anti-rabbit IgG secondary antibody (1:500; Zhongshan Golden Bridge Biotechnology, Beijing, China; ZLI-9022). The sections were processed using a standard biotin-avidin-horseradish peroxidase methodology. Diaminobenzidine tetrahydrochloride (Beijing Zhongshan Jinqiao Biotechnology Co., Ltd.) was used as the chromogen, and immunoreactivity appeared as brown staining. The nuclei were counterstained with hematoxylin. Sections were examined using light microscopy.

Statistical analysis. Data were expressed as the mean \pm standard error of the mean. Data were analyzed using SPSS 21 (IBM SPSS, Armonk, NY, USA) and GraphPad Prism 6

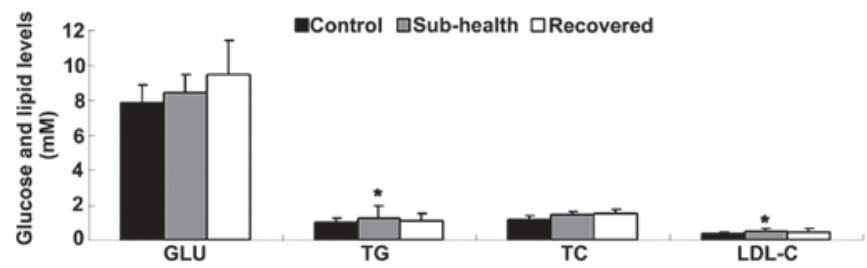

Figure 1. Effects of sub-health factors on the glucose and lipid profile. Data are presented as the mean \pm standard deviation. ${ }^{*} \mathrm{P}<0.05$, vs. control. GLU, glucose; TG, triglycerides; TC, total cholesterol; LDL-C, low density lipoprotein cholesterol.

(GraphPad Software, Inc., La Jolla, CA, USA). Comparisons between groups were analyzed with appropriate analyses of variance or t-tests. $\mathrm{P}<0.05$ was considered to indicate a statistically significant difference.

\section{Results}

Changes in growth, GLU and lipid profile. The present study initially observed that body weight of the rats in the sub-health group were similar to those of the rats in the control group (data not shown), indicating that chronic stress may affect the increase of weight regardless of a high-fat and sugar diet. As shown in Fig. 1, the GLU and lipid levels in the sub-health rats increased marginally, compared with the control rats, but without statistical significance. However, the levels of TGs and LDL-c were significantly upregulated in the sub-health rats, compared with the control group. In addition, in the recovered group, the level of GLU and the lipid profile did not return to normal.

Changes in IFN- $\gamma$ and IL-4 concentrations. The serum concentrations of IFN- $\gamma$ and IL-4 were significantly decreased in the sub-health rats, compared with the control group. Following recovery from the sub-health condition for 35 days, the levels of IFN- $\gamma$ and IL-4 remained unchanged in the recovered sub-health group (Fig. 2).

Behavioral investigation. The effects of the sub-health conditions on the number of crossing responses and rearing responses (motor activity), the duration spent in the central area (habituation) and fecal boli (emotionality) in the open field task are presented in Table I. An increased number of boli is associated with the anxiety of the animal (11-13). Statistical analysis demonstrated a significant reduction in the number of 
A

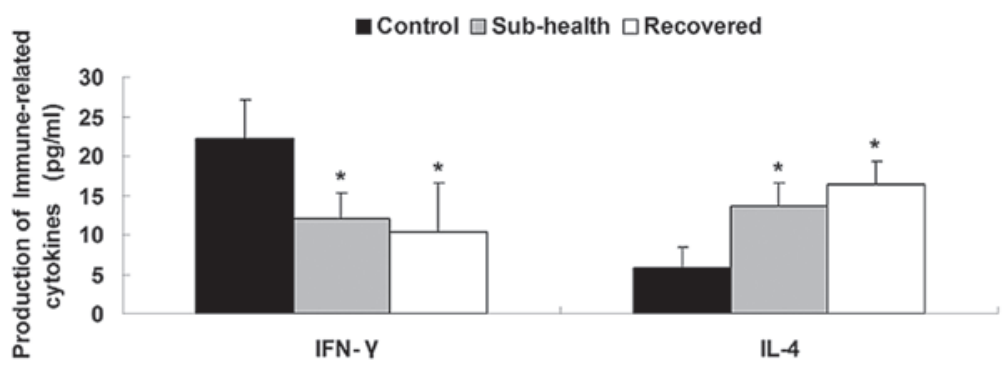

B

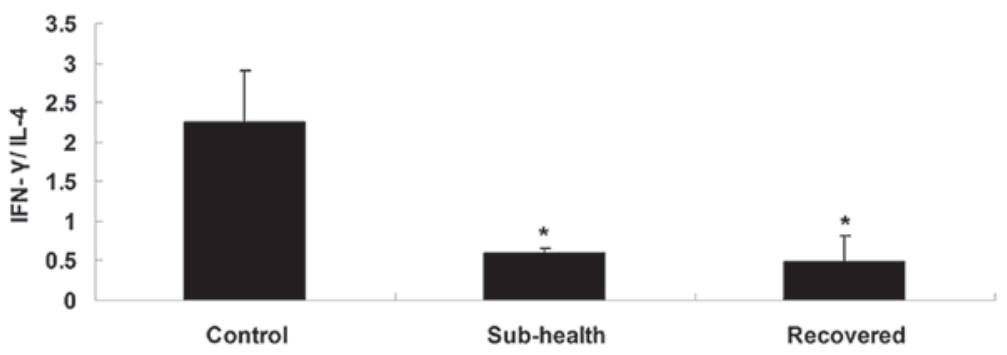

Figure 2. Production of immune-associated cytokines. (A) Serum level of IFN- $\gamma(\mathrm{pg} / \mathrm{ml})$ in the sub-health group and recovered group were lower than that in the control group, and the levels of IL-4 (pg/ml) in the sub-health group and recovered group were higher than that in the control group. (B) IFN- $\gamma / \mathrm{IL}-4$ ratios in the sub-health group and recovered group were lower than that in the control group. Data are presented as the mean \pm standard deviation. "P<0.05, vs. control. IFN, interferon; IL, interleukin.

A

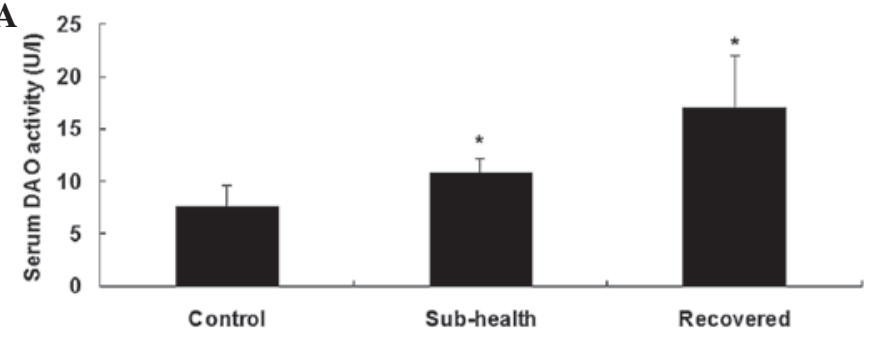

B

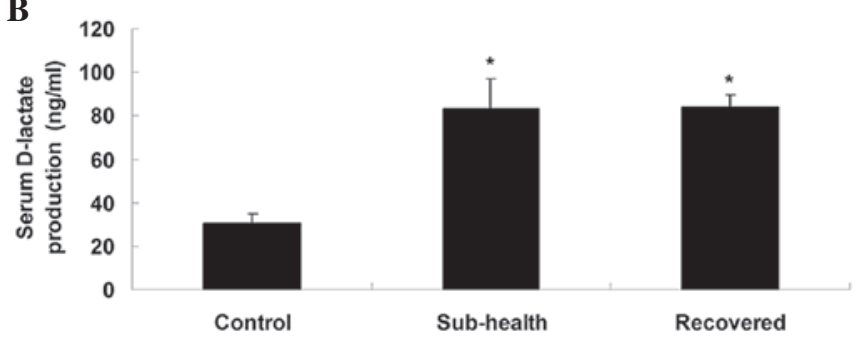

Figure 3. Serum levels of D-lactate and DAO. (A) Sub-health factors increased the level of D-lactate. (B) Levels of DAO were higher in the sub-health group, compared with the control group. Returning to normal conditions did not reduce the level of D-lactate or the activity of DAO. Data are presented as the mean \pm standard deviation. ${ }^{\mathrm{P}}<0.05$, vs. control. $\mathrm{DAO}$, diamine oxidase.
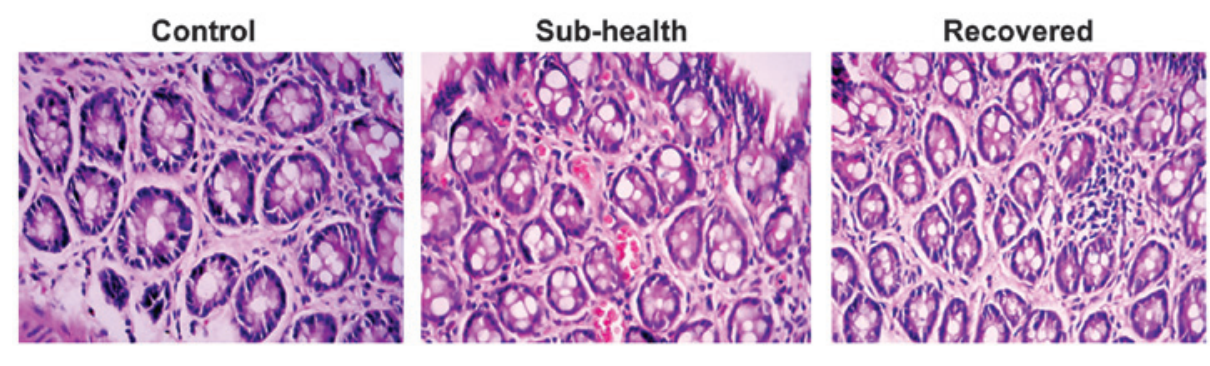

Figure 4. Changes in permeability are not due to altered colon architecture. Hematoxylin and eosin staining of colon tissue sections from rats demonstrated no difference in histological architecture and or cell proliferation between the control, sub-health and recovered groups. Magnification, x400.

crossings and rearings, and an increase in the time spent in the central area, indicating deficits in motor activity and habituation. The increase of fecal boli indicated that the sub-health factors also affected the emotionality of the animals. Following a return to normal living conditions, the behaviors of the rats improved marginally.

Changes in serum levels of D-lactic acid and the activity of $D A O$. The results of the present study revealed that the levels of serum D-lactate and the activity of DAO in the sub-health group were significantly higher, compared with those of the control group $(\mathrm{P}<0.001)$, however no significant change was observed in the levels of D-lactate and activity of DAO in the recovered group, compared with the sub-health group (Fig. 3).

Histological analyses. The histological architecture and cell proliferation in the colon tissues from the rats were evaluated following paraffin sectioning and hematoxylin and eosin 


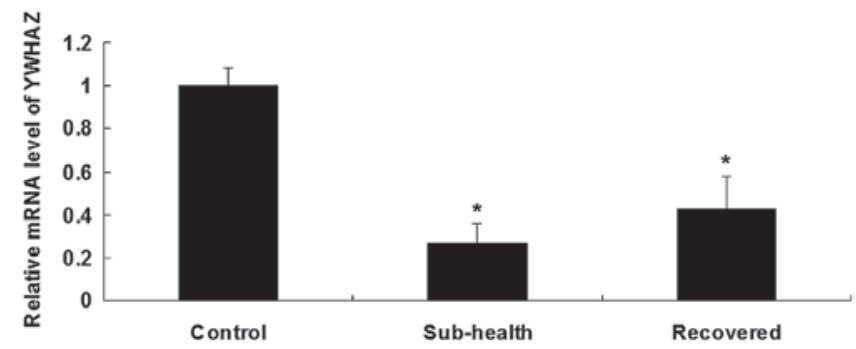

Figure 5. mRNA levels of YWHAZ were measured in the large intestine of rats using reverse transcription-quantitative polymerase chain reaction analysis. GAPDH was used as an internal reference. RNA was harvested from the rats subjected to sub-health factors at day 35 , and rats 35 days following return to their normal living patterns. Data are presented as the mean \pm standard deviation. ${ }^{*} \mathrm{P}<0.05$, vs. control.
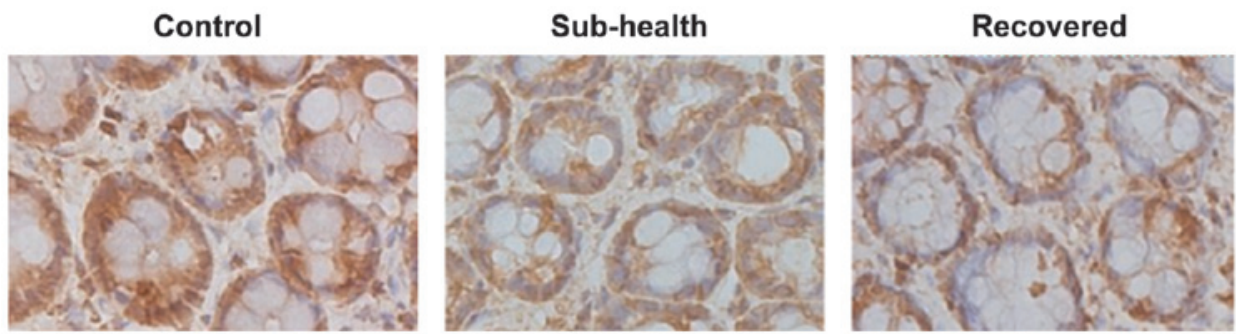

Figure 6. Immunohistochemical staining of YWHAZ. Decreased expression levels of YWHAZ were observed in the sub-health group and sub-health recovered group, compared with the control group. Magnification, $\mathrm{x} 400$.

staining. No significant differences were observed in the histology of the colon sections between the three groups (Fig. 4).

Change in the expression levels of YWHAZ. To investigate the molecular changes of barrier function damage in sub-health rats, the present study investigated the expression levels of YWHAZ. RT-qPCR revealed a significant decrease in the mRNA expression levels of YWHAZ in the rats treated with sub-health factors, indicating the inhibitory effect of sub-health factors on YWHAZ (Fig. 5). This suggested that sub-health factors contribute to the damage caused by the downregulation of YWHAZ. In addition, the immunohistochemical data revealed a significant decrease in the mRNA expression levels of YWHAZ in the rats treated with sub-health factors. Following 5 weeks recovery, the expression of YWHAZ remained at a low level (Fig. 6). These results indicated that the sub-health factors had an inhibitory effect on the expression of YWHAZ, and the return to normal living conditions does not affect the level of YWHAZ.

\section{Discussion}

In the present study, a novel sub-health rat model was established, which exhibited all the typical characteristics of sub-health, including high levels of glucose and lipids, weak immune function and deficits in activity, reaction and habituation. The present study also indicated that there was abnormal intestinal barrier function in the sub-health rat model. In addition, returning to normal living and feeding conditions did not improve mucosal barrier function.

Sub-health is defined by the World Health Organization as a state between health and disease, when all necessary physical and chemical indices have tested negative by medical equipment, however, the individual experiences discomfort and even pain (2). In the present study, due to being provided with a high-fat and sugar diet, the model rats had high levels of blood glucose and lipids, compared with the control group, however, the level did not confirm the presence of diabetes or hypercholesterolemia $(14,15)$. This is consistent with the characteristics of sub-health.

In sub-health conditions, the body has a weak immune system, and pathogens may invade easily with serious consequences. It has been established that exposure to various pathogens can stimulate at least two patterns of cytokine production by CD4-positive T cells (16). Responses, which result in the secretion of IFN- $\gamma$, lymphotoxin and IL-2 are classified as T-helper-1 (Th1 response, whereas $\mathrm{CD}^{4+} \mathrm{T}$-cell production of IL-4, IL-5, IL-9, IL-10 and IL-13 is termed a Th2 response (17). Differentiation of $\mathrm{CD}^{+} \mathrm{T}$ cells into either Th1 or Th2 cells is determined according to the cytokine milieu in which the initial antigen priming occurs. IFN- $\gamma$ is secreted at higher levels in splenic lymphocytes, which indicates that a Th1 response is evoked through immunization. Accordingly, the antigen-sensitized lymphocytes were present and activated under the effect of IFN- $\gamma$ (17). In addition, secretions of IL-4 at higher levels are involved in combination with the induction of $\operatorname{IgA}$ and the differentiation of B cells. Thus, the cytokines secreted are relative to cell-mediated immune responses and humoral immune responses $(18,19)$. The results of the present study revealed lower levels of IFN- $\gamma$ and IL- 4 in the model rats, compared with the control rats, indicating that weak immune function is also one of the characteristics of sub-health.

Lack of energy, depression, slow reactions and lack of interest or listlessness are common symptoms of sub-health. Open field assessment has been used widely to assess habituation, emotionality and locomotor performance $(20,21)$. Upon exposure to a standard open field central zone, rats move rapidly 
towards the walls of the field, a behavior known as thigmotaxis. This behavior represents the propensity to avoid the center of an arena and stay or move in close proximity to the boundaries or walls of a novel environment (22). Activity in the central part of the open field is considered to correlate with a degree of habituation, whereas activity in the peripheral zone and along the walls of the field is considered to reflect general activity (23). In the present study, the model rats exhibited a deficit in activity and habituation. In addition, the increase in the number of fecal boli indicated increased anxiety in the model rats. These characteristics of the model rats demonstrated that it was a suitable mode for investigating sub-health.

Mucosal surfaces of the gastrointestinal tract are continuously exposed to environmental stimuli. The intestinal epithelium constitutes the largest and most important barrier against external environmental agents and has two critical functions: To prevent the entry of harmful intraluminal microorganisms, antigens and toxins, and to enable the selective translocation of dietary nutrients and electrolytes into the circulation (24). Similar to irritable bowel syndrome, prolonged and high-intensity exercise, dietary fat and intestinal disorders in sub-health may also result from abnormal intestinal barrier function. DAO, an intracellular enzyme catalyzing the oxidation of diamines, including histamine, putrescine and cadaverine, exist in high concentrations in the intestinal mucosa, and the majority of DAO activity in the blood comes from the intestine (25). The serum activity of DAO is proportional to the level of intestinal DAO; and is a reliable marker of intestinal mucosal integrity (26). D-lactic acid is a metabolic product of bacteria, present in the intestinal lumen. The intact intestinal mucosa provides a barrier function to prevent DAO and D-lactic acid infiltrating the portal blood, which are indices of increase in permeability of the intestinal wall (27). In the present study the serum levels of DAO and D-lactic acid in the sub-health rats were enhanced, which indicated that sub-health factors increased the epithelial permeability, compromising the intestinal function. Improved mucosal barrier function may be a target of therapy in sub-health intestinal abnormality.

In our previous study (8), which aimed to identify the molecule marker of the progress of proteomics in the colonic mucosa of sub-healthy individuals, two-dimensional electrophoresis was used to separate the total colonic mucosa proteins of sub-healthy individuals and those of healthy volunteers, and further confirmation of differentially expressed proteins was performed using western blot analysis. It was found that the expression of YWHAZ was downregulated in the colonic mucosa, which may be associated with the pathogenesis of abnormal intestinal function. YWHAZ is important role in a wide range of biologic processes through a variety of regulatory mechanisms, by binding to phosphorylated serine/threonine motifs in diverse partners, the most important of which is its inhibitory effect on apoptosis $(28,29)$. Apoptosis can lead to defects in intestinal barrier function, and the maintenance of intestinal function is reliant on the rapid renewal of the intestinal mucosa and cell proliferation $(30,31)$. Therefore, the downregulation in the expression of YWHAZ in the colonic mucosa of sub-health rats may be involved in compromised mucosa barrier function through the upregulation of apoptosis and inhibition of cell proliferation.
There remains no consensus on whether sub-health necessitates drug-based intervention. It has been suggested that isolating and removing the causes of sub-health, as well as environmental or psychological factors, would cause sub-health symptoms to disappear (4). In the present study, following rescue from pressure and limited space for 5 weeks, the adaptability and vitality of the sub-health rats exhibited certain improvements. However, the levels of serum glucose and lipids, as well as the immune function, did not recover. In addition the intestinal mucosal permeability and the protein expression of YWHAZ remained abnormal. Therefore, other interventions may be necessary, possibly including fish oil supplementation, exercise, music therapy and probiotics, which may be useful (32-34).

\section{References}

1. World Health Organization: Preamble to the constitution of the World Health Organization as adopted by the International Health Conference, New York, 19-22 June, 1946; signed on 22 July 1946 by the representatives of 61 states. Official Record of the World Health Organization 2: 100, 1946.

2. Yang ZM, Yang XB and Huang L: A literature review on the conceptual framework of sub-health. Zhongguo Zhong Xi Yi Jie He Za Zhi 30: 757-763, 2010 (In Chinese).

3. Zhang YJ, Wang TF, Xue XL, Wang JJ, Li GR and Han P: Characteristics of traditional Chinese medicine syndromes and their element distributions in sub-health status: A modern literature review. Zhong Xi Yi Jie He Xue Bao 6: 1290-1293, 2008 (In Chinese).

4. Zhou Y, You LM, Zhang JB and Guan NH: Causes and prevention of sub-health. Chinese Journal of Health Education 18: 714-715, 2002 (In Chinese).

5. Xing L: Risk factors and nursing intervention of sub-health. Medical Information 28: 206, 2015 (In Chinese).

6. Vazquez-Roque MI, Camilleri M, Smyrk T, Murray JA, O'Neill J, Carlson P, Lamsam J, Eckert D, Janzow D, Burton D, et al: Association of HLA-DQ gene with bowel transit, barrier function and inflammation in irritable bowel syndrome with diarrhea. Am J Physiol Gastrointest Liver Physiol 303: G1262-G1269, 2012.

7. Zuhl M, Schneider S, Lanphere K, Conn C, Dokladny K and Moseley P: Exercise regulation of intestinal tight junction proteins. Br J Sports Med 48: 980-986, 2014.

8. Deng H, Zhang YL, Zhong BY, Liu WD, Yan Q, Feng SS and Xiao ZQ: Clinical application and identification of proteomics in colonic mucosa of sub-health people with constipation. Progress in Biochemistry and Biophysics 11: 1043-1051, 2011 (In Chinese).

9. Million M, Taché Y and Anton P: Susceptibility of Lewis and Fischer rats to stress-induced worsening of TNB-colitis: Protective role of brain CRF. Am J Physiol 276: G1027-G1036, 1999.

10. Csabafi K, Jászberényi M, Bagosi Z, Lipták N and Telegdy G: Effects of kisspeptin-13 on the hypothalamic-pituitary-adrenal axis, thermoregulation, anxiety and locomotor activity in rats. Behav Brain Res 241: 56-61, 2013.

11. Hall CS: Emotional behavior in the rat. I. Defacation and urination as measures of individual differences in emotionality. J Comp Psychol 18: 385-403, 1934.

12. Whimbey AE and Denenberg VH: Two independent behavioral dimensions in open-field performance. J Comp Physiol Psychol 63: 500-504, 1967

13. Flint J, Corley R, DeFries JC, Fulker DW, Gray JA, Miller S and Collins AC: A simple genetic basis for a complex psychological trait in laboratory mice. Science 269: 1432-1435, 1995.

14. Toker H, Ozdemir H, Balci H and Ozer H: N-acetylcysteine decreases alveolar bone loss on experimental periodontitis in streptozotocin-induced diabetic rats. J Periodontal Res 47: 793-799, 2012.

15. Füzi M, Palicz Z, Vincze J, Cseri J, Szombathy Z, Kovács I, Oláh A, Szentesi P, Kertai P, Paragh G and Csernoch L: Fluvastatin-induced alterations of skeletal muscle function in hypercholesterolaemic rats. J Muscle Res Cell Motil 32: 391-401, 2012.

16. Luo J and Huang L: Research progress in the identification and classification of sub-health state. Chinese Medicine Modern Distance Education of China 13: 154-156, 2015 (In Chinese). 
17. Ferrick DA, Schrenzel MD, Mulvania T, Hsieh B, Ferlin WG and Lepper H: Differential production of interferon-gamma and interleukin-4 in response to Th1- and Th2-stimulating pathogens by gamma delta T cells in vivo. Nature 373: 255-257, 1995.

18. Yao XY, Wang HM, Li DJ, Yuan MM, Wang XL, Yu M, Wang MY, Zhu Y and Meng Y: Inoculation of Lactobacillus expressing hCG beta in the vagina induces an anti-hCG beta antibody response in murine vaginal mucosa. J Reprod Immunol 63: 111-122, 2004.

19. Liu G,Zhong J, Ni J, Chen M, Xiao H and Huan L: Characteristics of the bovicin HJ50 gene cluster in Streptococcus bovis HJ50. Microbiology 155: 584-593, 2009.

20. Choleris E, Thomas AW, Kavaliers M and Prato FS: A detailed ethological analysis of the mouse open field test: Effects of diazepam, chlordiazepoxide and an extremely low frequency pulsed magnetic field. Neurosci Biobehav Rev 25: 235-260, 2001.

21. Prut $\mathrm{L}$ and Belzung $\mathrm{C}$ : The open field as a paradigm to measure the effects of drugs on anxiety-like behaviors: A review. Eur J Pharmacol 463: 3-33, 2003.

22. Sharma S, Coombs S, Patton P and Burt de Perera T: The function of wall-following behaviors in the Mexican blind cavefish and a sighted relative, the Mexican tetra (Astyanax). J Comp Physiol A Neuroethol Sens Neural Behav Physiol 195: 225-240, 2009.

23. Belzung $C$ and Griebel G: Measuring normal and pathological anxiety-like behaviour in mice: A review. Behav Brain Res 125 141-149, 2001.

24. McGhee JR and Fujihashi K: Inside the mucosal immune system. PLoS Biol 10: e1001397.

25. Kamiya S, Nagino M, Kanazawa H, Komatsu S, Mayumi T, Takagi K, Asahara T, Nomoto K, Tanaka R and Nimura Y: The value of bile replacement during external biliary drainage: An analysis of intestinal permeability, integrity and microflora. Ann Surg 239: 510-517, 2004.
26. Luk GD, Bayless TM and Baylin SB: Plasma postheparin diamine oxidase. Sensitive provocative test for quantitating length of acute intestinal mucosal injury in the rat. J Clin Invest 71: 1308-1315, 1983.

27. Smith SM, Eng RH, Campos JM and Chmel H: D-lactic acid measurements in the diagnosis of bacterial infections. J Clin Microbiol 27: 385-388, 1989.

28. Gardino AK and Yaffe MB: 14-3-3 proteins as signaling integration points for cell cycle control and apoptosis. Semin Cell Dev Biol 22: 688-695, 2011.

29. Masters SC and $\mathrm{Fu} \mathrm{H}$ : 14-3-3 proteins mediate an essential anti-apoptotic signal. J Biol Chem 276: 45193-45200, 2001.

30. Bharadwaj S, Vishnubhotla R, Shan S, Chauhan C, Cho M and Glover SC: Higher molecular weight polyethylene glycol increases cell proliferation while improving barrier function in an in vitro colon cancer model. J Biomed Biotechnol 2011: 587470, 2011.

31. Bojarski C, Bendfeldt K, Gitter AH, Mankertz J, Fromm M, Wagner S, Riecken EO and Schulzke JD: Apoptosis and intestinal barrier function. Ann N Y Acad Sci 915: 270-274, 2000.

32. Rachetti AL, Arida RM, Patti CL, Zanin KA, Fernades-Santos L, Frussa-Filho R, Gomes da Silva S, Scorza FA and Cysneiros RM: Fish oil supplementation and physical exercise program: Distinct effects on different memory tasks. Behav Brain Res 237: 283-289, 2013.

33. Jirillo E, Jirillo F and Magrone T: Healthy effects exerted by prebiotics, probiotics and symbiotics with special reference to their impact on the immune system. Int J Vitam Nutr Res 82: 200-208, 2012

34. Korhan EA, Uyar M, Eyigör C, Hakverdioğlu Yönt G, Çelik S and Khorshid L: The effects of music therapy on pain in patients with neuropathic pain. Pain Manag Nurs 15: 306-314, 2014. 